\title{
A scaled MP-PIC method for bubbling fluidization
}

\author{
Xing Zhao ${ }^{1}$, Yong Jiang ${ }^{1}$, Fei $\mathrm{Li}^{1}$, and Wei Wang ${ }^{1}$ \\ ${ }^{1}$ Institute of Process Engineering Chinese Academy of Sciences
}

September 25, 2021

\begin{abstract}
Coarse-grained methods have been widely used in simulations of gas-solid fluidization. However, as a key parameter, the coarsegraining ratio, and its relevant scaling law is still far from reaching a consensus. In this work, a scaling law is developed based on a similarity analysis, and then it is used to scale the multi-phase particle-in-cell (MP-PIC) method, and validated in the simulation of two bubbling fluidized beds. The simulation result shows this scaled MP-PIC can reduce the errors of solids volume fraction and velocity distributions over a wide range of coarse-graining ratios. In future, we expect that a scaling law with consideration of the heterogeneity inside a parcel or numerical particle will further improve the performance of coarse-grained modeling in simulation of fluidized beds.
\end{abstract}

\section{Hosted file}

main-aiche.pdf available at https://authorea.com/users/436207/articles/538701-a-scaled-mppic-method-for-bubbling-fluidization

\section{Hosted file}

fig 1.pptx available at https://authorea.com/users/436207/articles/538701-a-scaled-mp-picmethod-for-bubbling-fluidization

\section{Hosted file}

fig 2.pptx available at https://authorea.com/users/436207/articles/538701-a-scaled-mp-picmethod-for-bubbling-fluidization

\section{Hosted file}

fig 3.pptx available at https://authorea.com/users/436207/articles/538701-a-scaled-mp-picmethod-for-bubbling-fluidization

\section{Hosted file}

fig 4.pptx available at https://authorea.com/users/436207/articles/538701-a-scaled-mp-picmethod-for-bubbling-fluidization

\section{Hosted file}

fig 5.pptx available at https://authorea.com/users/436207/articles/538701-a-scaled-mp-picmethod-for-bubbling-fluidization

\section{Hosted file}

fig 6.pptx available at https://authorea.com/users/436207/articles/538701-a-scaled-mp-picmethod-for-bubbling-fluidization

\section{Hosted file}


fig 7.pptx available at https://authorea.com/users/436207/articles/538701-a-scaled-mp-picmethod-for-bubbling-fluidization

\section{Hosted file}

fig 8.pptx available at https://authorea.com/users/436207/articles/538701-a-scaled-mp-picmethod-for-bubbling-fluidization

\section{Hosted file}

fig 9.pptx available at https://authorea.com/users/436207/articles/538701-a-scaled-mp-picmethod-for-bubbling-fluidization

\section{Hosted file}

fig 10.pptx available at https://authorea.com/users/436207/articles/538701-a-scaled-mp-picmethod-for-bubbling-fluidization

\section{Hosted file}

fig C1.pptx available at https://authorea.com/users/436207/articles/538701-a-scaled-mp-picmethod-for-bubbling-fluidization

\section{Hosted file}

fig C2.pptx available at https://authorea.com/users/436207/articles/538701-a-scaled-mp-picmethod-for-bubbling-fluidization

\section{Hosted file}

Table. 1 Scaling laws for coarse-grained models in literature.docx available at https: //authorea.com/users/436207/articles/538701-a-scaled-mp-pic-method-for-bubblingfluidization

\section{Hosted file}

Table. 2 The characteristic quantities adopted in dimensionless equation.docx available at https://authorea.com/users/436207/articles/538701-a-scaled-mp-pic-method-for-bubblingfluidization

\section{Hosted file}

Table. 3 Sample particle parameters in original and scaled MP-PIC.docx available at https://authorea.com/users/436207/articles/538701-a-scaled-mp-pic-method-for-bubblingfluidization

\section{Hosted file}

Table. 4 Numerical settings for bubbling bed 165 and bed 266.docx available at https: //authorea.com/users/436207/articles/538701-a-scaled-mp-pic-method-for-bubblingfluidization 